\title{
Hepatic Eosinophilic Abscess Associated with Sigmoid Colon Cancer
}

\author{
Yasuyuki Shigematsu ${ }^{1,2}$, Hiroaki Kanda ${ }^{1}$, Toshiya Nagasaki ${ }^{3}$, Takeaki Ishizawa ${ }^{3}$, \\ Yosuke Inoue $^{3}$ and Shunji Takahashi ${ }^{2}$
}

\begin{abstract}
:
The clinical course of hepatic eosinophilic abscess (HEA) induced by malignant tumors is not well-known; however, it is considered to be a benign hepatic lesion. HEA is difficult to diagnose by imaging alone and a pathological examination is generally needed, particularly in patients with malignant tumors, because the radiological findings can be similar to those of metastasis. We report a case of multiple HEAs with eosinophilia and sigmoid colon cancer that was difficult to diagnose without a pathological examination. After the resection of the sigmoid colon cancer, the patient's eosinophilia was quickly ameliorated and the HEAs disappeared within 6 months.
\end{abstract}

Key words: hepatic eosinophilic abscess, colon cancer, eosinophilia

(Intern Med 57: 1405-1409, 2018)

(DOI: 10.2169/internalmedicine.0083-17)

\section{Introduction}

Eosinophilic organ infiltration can sometimes occur in patients with eosinophilia, it damages target organs, and may result in abscess formation (1). Eosinophilia and eosinophilic organ infiltration are known to be induced by parasitic infection, drug hypersensitivity, allergic disease, collagen vascular disease, and malignant tumors (2). Irrespective of the cause of the eosinophilic infiltration, the target organs can be the skin, lungs, liver, gastrointestinal tract, heart, and central nervous system $(3,4)$. The frequency at which these organs are involved and the severity of the disease varies according to the cause.

Hepatic eosinophilic abscess (HEA) can be characterized by localized eosinophilic infiltration near the portal vein with or without central necrosis in the liver (5). The clinical course of HEA is considered to be benign after the underlying cause of the eosinophilia is corrected; however, in clinical practice, the underlying causes are often difficult to identify (6). There are limited data on the clinical characteristics, pathophysiology, and clinical course of the eosinophilic abscesses induced by solid malignant tumors.
The liver is one of the common target organs of eosinophils. A diagnosis of HEA is difficult to make in cancer patients because the imaging features can be similar to those of metastasis; thus, it can be difficult to differentiate between eosinophilic abscesses and metastatic lesions on the basis of radiologic findings alone $(7,8)$.

We experienced an asymptomatic case with multiple HEAs coexisting with eosinophilia and sigmoid colon cancer. In this case, after the resection of the sigmoid colon cancer, the eosinophilia and the HEAs (which had been pathologically confirmed) disappeared within 6 months. We herein report the findings of the present case, which featured a unique clinical scenario of eosinophilia and multiple HEAs that developed in association with sigmoid colon cancer.

\section{Case Report}

A 59-year-old asymptomatic Japanese man visited our hospital due to a positive fecal occult blood test. His medical history showed that he had experienced one episode of gastric ulcer at 30 years of age. He regularly used a number of medications, including valsartan, amlodipine, and atorvas-

${ }^{1}$ Department of Pathology, The Cancer Institute of Japanese Foundation for Cancer Research (JFCR), Japan, ${ }^{2}$ Division of General Oncology, The Cancer Institute Hospital of JFCR, Japan and ${ }^{3}$ Division of Gastroenterology Center, The Cancer Institute Hospital, JFCR, Japan Received for publication August 15, 2017; Accepted for publication September 20, 2017 Correspondence to Dr. Yasuyuki Shigematsu, yasuyuki.shigematsu@jfcr.or.jp 
tatin, for hypertension and hyperlipidemia. He did not have any allergies. At the first visit, his blood pressure, pulse rate, and body temperature were 133/92 $\mathrm{mmHg}, 70$ beats per minute, and $36.8^{\circ} \mathrm{C}$, respectively, and he seemed to be fine. A physical examination revealed no abnormal findings. The laboratory data showed slight eosinophilia and normal serum chemistry levels (Table). Lower gastrointestinal endoscopy revealed the presence of a $17-\mathrm{mm}$ sessile tumor in the sigmoid colon. A histopathological examination of the biopsied tumor revealed well-differentiated tubular adenocarcinoma. Contrast-enhanced computed tomography (CT) of the whole

Table. Blood Test before the Surgery.

\begin{tabular}{lc}
\hline WBC & $6,300 / \mu \mathrm{L}$ \\
Neutrophil & $37.1 \%$ \\
Lymphocyte & $42.4 \%$ \\
Monocyte & $11.5 \%$ \\
Eosinophil & $8.1 \%$ \\
Hemoglobin & $15.4 \mathrm{~g} / \mathrm{dL}$ \\
Plt & $25.9 \times 10^{4} / \mu \mathrm{L}$ \\
AST & $22 \mathrm{U} / \mathrm{L}$ \\
ALT & $32 \mathrm{U} / \mathrm{L}$ \\
ALP & $191 \mathrm{U} / \mathrm{L}$ \\
T.Bil & $0.4 \mathrm{mg} / \mathrm{dL}$ \\
ALB & $4.3 \mathrm{~g} / \mathrm{dL}$ \\
BUN & $12 \mathrm{mg} / \mathrm{dL}$ \\
Creatinin & $0.62 \mathrm{mg} / \mathrm{dL}$ \\
CRP & $0.09 \mathrm{mg} / \mathrm{dL}$ \\
CEA & $1.8 \mathrm{ng} / \mathrm{mL}$ \\
CA19-9 & $3.3 \mathrm{U} / \mathrm{mL}$ \\
\hline
\end{tabular}

WBC: white blood cells, Plt: platelets, AST: aspartate aminotransferase, ALT: alanine transaminase, ALP: alkaline phosphatase, T.Bil: total bilirubin, ALB: albumin, BUN: blood urea nitrogen, CRP: C-reactive protein, CEA: carcinoembryonic antigen, CA19-9: carbohydrate antigen 19-9 body, from the chest to the pelvic region, showed multiple irregular patchy lesions in the right hepatic lobe. All of the lesions showed early-phase contrast enhancement and some also showed a relatively low-density area in the central region (Fig. 1A and $\mathrm{B}$ ). There also appeared to be highdensity areas in the late-phase of contrast enhancement (Fig. 1C). Gadoxetic-acid-enhanced magnetic resonance imaging (MRI) of the abdomen provided little additional information because the intensity showed little variation between the tumor-like and non-tumor regions. In addition, image modification occurred because of fatty liver changes. An abdominal ultrasound examination revealed that the lesions were hypoechoic; however, the images were not sufficient for determining the diagnosis of the liver lesions. From these findings, the possibility of liver metastasis of sigmoid colon cancer could not be ruled out. Thus, laparoscopic sigmoid colectomy with D3 lymph node (LN) dissection was performed for the treatment of sigmoid colon cancer and one of the hepatic lesions was partially resected for diagnostic purposes.

The pathological examination of the sigmoid colon cancer revealed that the cancer cells had invaded the submucosa with massive vascular involvement. No cancer cells were found in the 25 dissected LNs (pT1N0M0, UICC 7th Stage I; Fig. 2). A well-circumscribed mass lesion of $10 \mathrm{~mm}$ in diameter and with surfaces that appeared white or yellow was detected in the liver (Fig. 3A). The histopathological examination of the nodules was characterized by dense inflammatory infiltration with prominent eosinophils and necrotic lesions surrounded by epithelioid histiocytes (Fig. 3B and C). Obliterative phlebitis was observed in and around the abscesses (Fig. 3D and E). The background showed diffuse hepatic steatosis and eosinophilic infiltration along the portal veins (Fig. 3F).

After the resection of the sigmoid colon cancer, the eosinophil count suddenly returned to a normal level (Fig. 4). No special treatment was provided for the patient's

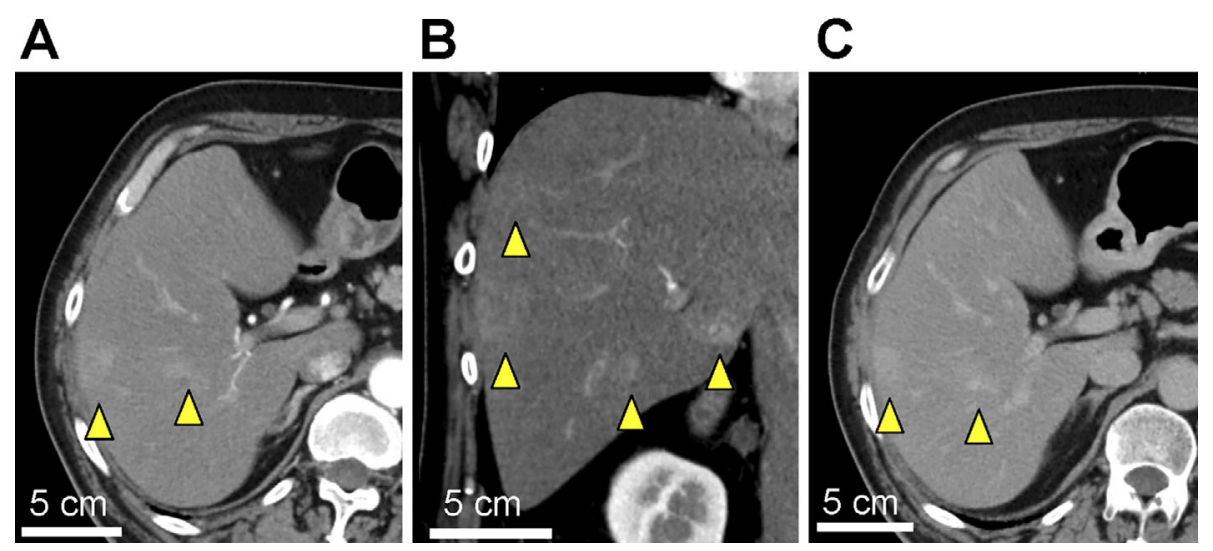

Figure 1. Contrast-enhanced computed tomography (CT) of the abdomen at admission. (A) An axial view and (B) a coronal view in the arterial phase. (A, B) Multiple enhanced lesions (yellow arrows) were observed in the liver. The borders of the lesions were irregular and some had central lowdensity areas. (C) An axial view in the delayed phase. The lesions were uniformly enhanced. It was not possible to exclude the possibility of colon cancer metastasis based on these findings. 
A

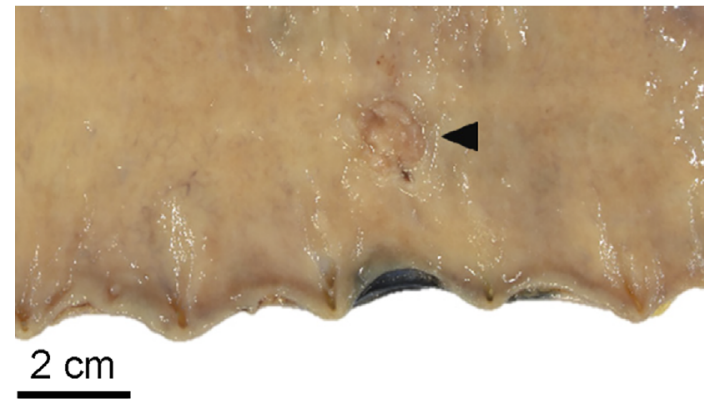

B

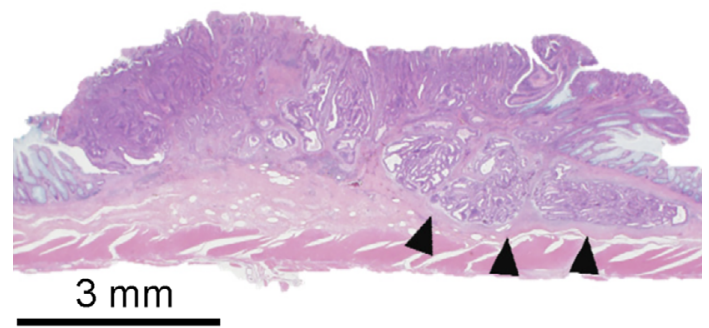

Figure 2. The pathological findings of the sigmoid colon cancer. (A) A macroscopic image shows a superficial elevated type tumor (black arrows; size $15 \times 12 \mathrm{~mm}$ ). (B) Victoria blue, Hematoxylin and Eosin staining of the tumor shows well-differentiated adenocarcinoma with submucosal invasion and massive venous invasion (black arrows).

HEAs. Six months later, contrast-enhanced CT showed that the remaining HEAs had also disappeared (Fig. 5A and B). Thus, the eosinophilia and the HEAs, which were observed during the patient's initial visit to our hospital, were considered to be associated with his sigmoid colon cancer. The patient performance status remains good and no disease recurrence has been detected in the 1 year since surgery.

\section{Discussion}

We reported a case of multiple HEAs associated with sigmoid colon cancer. In this case, the HEAs, which were pathologically confirmed, disappeared within 6 months after the resection of the sigmoid colon cancer. The overall incidence of eosinophilia in patients with malignant tumors is reported to be $12.6 \%$, while that in healthy individuals people is reported to be $6 \%$ (9). The incidence depends on the type of cancer (10). The highest incidence of eosinophilia is reported in Hodgkin lymphoma, followed by non-Hodgkin lymphoma and cervical carcinoma (9). The incidence was considered to be positively associated with tumor progression (9). To the best of our knowledge, this is the first case in which eosinophilia and HEAs coexisted with early-stage colon cancer. Few previous reports that have focused on the clinical course of HEAs induced by malignant tumors, especially in early-stage cancer. Thus, this case reflects a unique

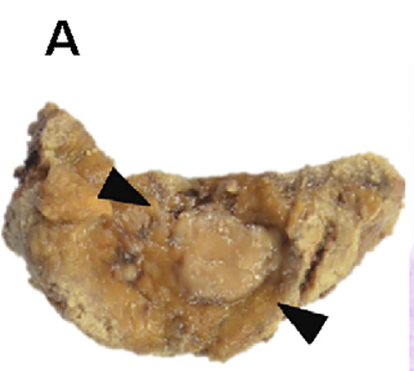

B
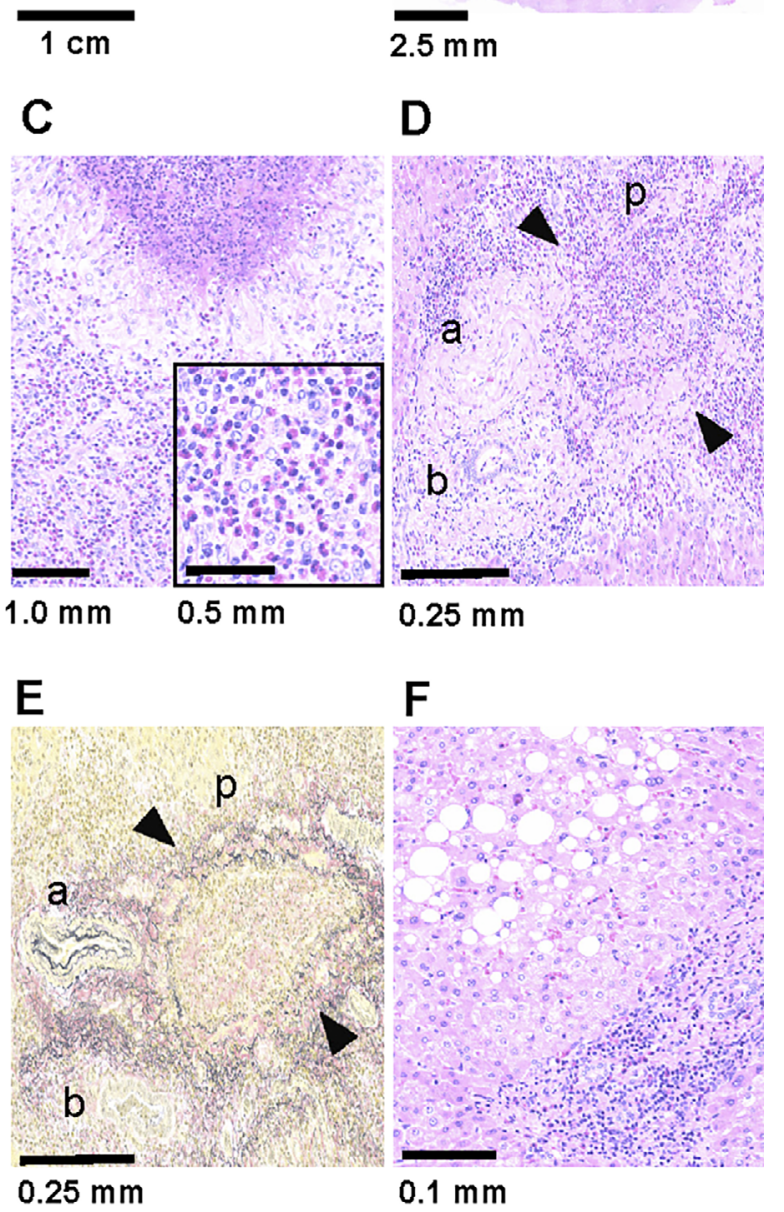

Figure 3. The pathological findings of the liver lesion in segment 8. (A) The macroscopic image indicates that the hepatic lesion (black arrows) was a homogeneous lesion that was mahogany in color. (B) Hematoxylin and Eosin (H\&E) staining of the hepatic lesion. The legion had several necrotic regions and an irregular border without a capsule. (C) Eosinophilic-rich granuloma with a palisade of epithelioid histiocytes. (D) H\&E staining and (E) Elastica van Gieson staining of the portal region. (D, E) The black arrows indicate obliterative phlebitis. The letters "a", "b", and " $p$ " indicate the "interlobular artery", "interlobular bile duct", and "interlobular portal vein", respectively. (F) In the background liver, diffuse hepatic steatosis and eosinophilic and lymphocytic infiltration were also observed along the portal veins.

\section{clinical course.}

Although the diagnosis of HEA was made without a pathological examination in some previous re- 


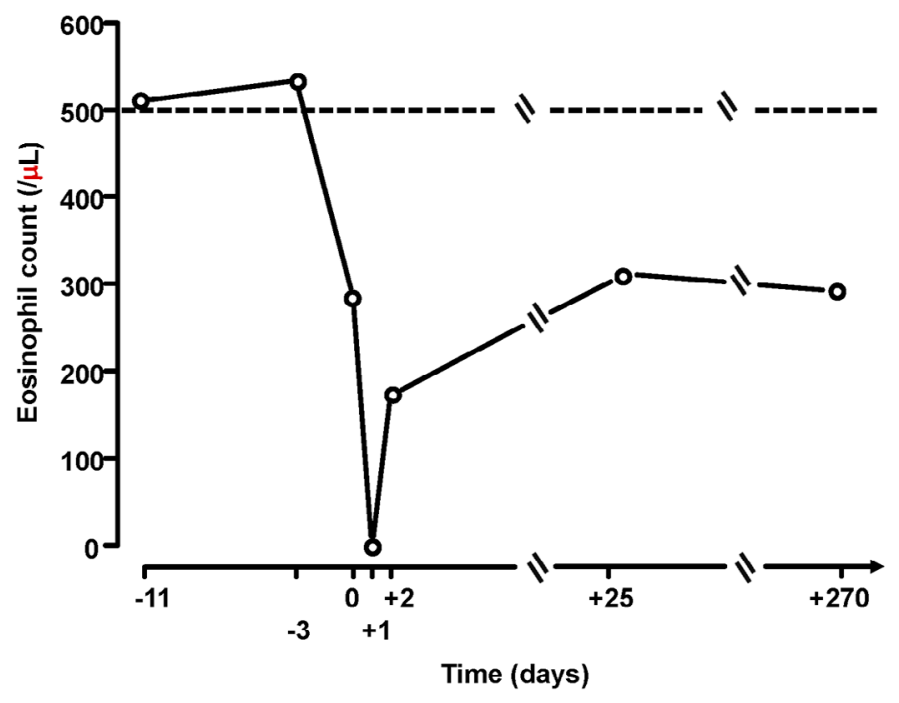

Figure 4. Changes in the eosinophil count over the clinical course. Day zero indicates the date of surgery. The eosinophil counts dramatically decreased after surgery and have since remained in the normal range.
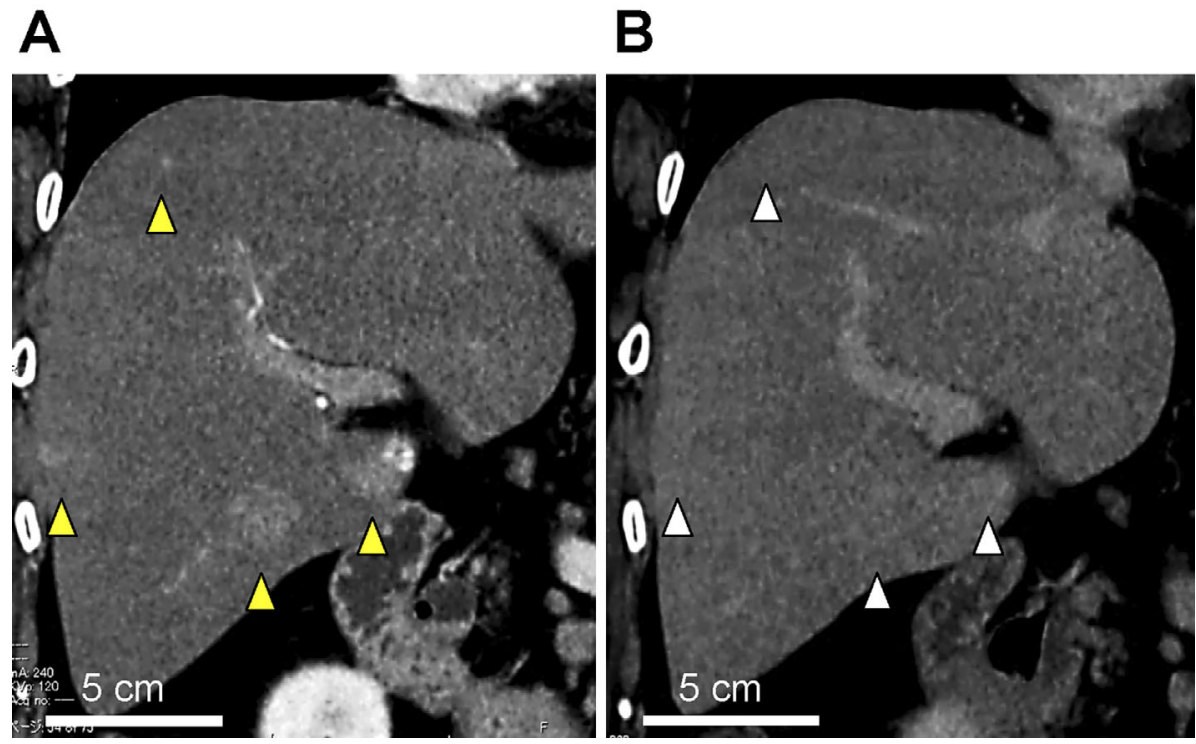

Figure 5. Changes in the hepatic lesions on contrast enhanced CT before and after the resection of the sigmoid colon cancer. The enhanced lesions (yellow arrows), which represented HEAs were observed before surgery, but were not observed (white arrows) on contrast-enhanced CT at the 6-month follow-up examination (B).

ports $(8,11,12)$, the clinical and imaging findings of HEAs are nonspecific and pathological confirmation is desirable (13-15). In this case, the findings of imaging modalities such as contrast-enhanced CT, contrast-enhanced MRI, and ultrasonography, were not sufficient for distinguishing the liver lesions from colon cancer metastasis. Laboratory studies also showed nonspecific findings aside from mild eosinophilia; thus, a pathological examination was required to confirm the diagnosis.

One mechanism through which tumor-associated HEAs develop in patients with underlying malignancy is as follows: the primary cancer cells produce eosinophilic chemo- tactic factors and the eosinophilic chemotactic factors are transported into the liver through portal flow and eosinophils are aggregated in the liver (5). In the primary colon cancer of our patient, the eosinophilic infiltration was mild and no eosinophilic abscess was observed. Although the sigmoid colon cancer was in the early-stage, the cancer had massive venous invasion, which possible induced eosinophilia and the HEAs might have affected the target organs through the blood vessels.

In conclusion, we experienced a case with a unique clinical course of multiple HEAs that coexisted with sigmoid colon cancer. After the resection of the sigmoid colon cancer, 
the eosinophilia was quickly ameliorated and the multiple HEAs disappeared within 6 months.

The authors state that they have no Conflict of Interest (COI).

\section{Acknowledgement}

We thank Mr. Motoyoshi Iwakoshi, Ms. Tomoyo Kakita, and Ms. Kimiyo Kogure for their kind assistance in the sample preparation.

\section{References}

1. Kwon JW, Kim TW, Kim KM, et al. Clinical features of clinically diagnosed eosinophilic liver abscesses. Hepatol Int 5: 949-954, 2011.

2. Kovalszki A, Weller PF. Eosinophilia. Prim Care 43: 607-617, 2016.

3. Roufosse F, Weller PF. Practical approach to the patient with hypereosinophilia. J Allergy Clin Immunol 126: 39-44, 2010.

4. Tefferi A. Blood eosinophilia: a new paradigm in disease classification, diagnosis, and treatment. Mayo Clin Proc 80: 75-83, 2005.

5. You SH, Park BJ, Kim YH. Hepatic lesions that mimic metastasis on radiological imaging during chemotherapy for gastrointestinal malignancy: recent updates. Korean J Radiol 18: 413-426, 2017.

6. Gotlib J, Cross NCP, Gilliland DG. Eosinophilic disorders: molecular pathogenesis, new classification, and modern therapy. Best Pract Res Clin Haematol 19: 535-569, 2006.

7. Jackson G, Kathuria M, Abraham B, Schnadig VJ. Fine needle aspiration diagnosis of necrotizing eosinophilic abscess clinically mimicking hepatic neoplasia: a case report. Acta Cytol 54: 60-62,
2010.

8. Lee T, Lee YS, Yoon SY, et al. Clinical characteristics that distinguish eosinophilic organ infiltration from metastatic nodule development in cancer patients with eosinophilia. World J Surg Oncol BioMed Central 10: 175, 2012.

9. Kumar H, Sivadas P, Rangarao R, Bhardwaj JR. Tumour associated blood eosinophilia. Med J Armed Forces India 51: 75-76, 1995.

10. Lowe D, Jorizzo J, Hutt MS. Tumour-associated eosinophilia: a review. J Clin Pathol 34: 1343-1348, 1981.

11. Gao SJ, Wei W, Chen JT, et al. Hypereosinophilic syndrome presenting with multiple organ infiltration and deep venous thrombosis: a case report and literature review. Medicine 95: e4658, 2016.

12. Kwon JW, Kim TW, Kim KM, et al. Clinical features of clinically diagnosed eosinophilic liver abscesses. Hepatol Int 5: 949-954, 2011.

13. Kwak HS, Han YM, Lee JM. Focal eosinophilic infiltration of the liver mimicking hepatocellular carcinoma. Clin Imaging 28: 36-39, 2004.

14. Won JH, Kim MJ, Kim BM, et al. Focal eosinophilic infiltration of the liver: a mimick of hepatic metastasis. Abdom Imaging 24: 369-372, 1999.

15. Byun JH, Yang DH, Yoon SE, et al. Contrast-enhancing hepatic eosinophilic abscess during the hepatic arterial phase: a mimic of hepatocellular carcinoma. AJR Am J Roentgenol 186: 168-173, 2006.

The Internal Medicine is an Open Access article distributed under the Creative Commons Attribution-NonCommercial-NoDerivatives 4.0 International License. To view the details of this license, please visit (https://creativecommons.org/licenses/ by-nc-nd/4.0/).

(C) 2018 The Japanese Society of Internal Medicine Intern Med 57: 1405-1409, 2018 